\title{
Rede de suporte social do idoso atendido por equipes de Saúde da Família
}

\author{
Social support networks for elderly patients \\ attended by Family Health teams
}

Márcia Regina Martins Alvarenga ${ }^{1}$

Maria Amélia de Campos Oliveira ${ }^{2}$

Marisa Accioly Rodrigues Domingues ${ }^{3}$

Fernanda Amendola ${ }^{2}$

Odival Faccenda ${ }^{1}$

${ }^{1}$ Universidade Estadual de Mato Grosso do Sul. Rodovia Dourados/Itahum $\mathrm{km} \mathrm{12,} \mathrm{caixa} \mathrm{postal} \mathrm{351,}$ Cidade Universitária. 79804-970 Dourados MS marciaregina@uems.br

${ }^{2}$ Escola de Enfermagem,

Universidade de São Paulo.

${ }^{3}$ Escola de Artes, Ciências e Humanidades,

Universidade de São Paulo.
Abstract The aging process has specific aspects marked by the class of individuals and social groups as well as cultural, political, socio-econom$i c$ and sanitary conditions of the collective groups. Social support systems are essential for meeting the specific needs of the elderly. The aim of this paper is to describe the socio-demographic profile and the social support networks of elderly patients served by the Family Health Strategy. It's a crosssectional study with elderly patients living in Dourados, in the state of Mato Grosso do Sul (Brazil). Data were collected using a socio-demographic questionnaire and a Minimum Relationships Map for the Elderly (MMRI). Of the 503 elderly patients interviewed, 69\% were female, $53.1 \%$ were illiterate, $58.3 \%$ earned less than one minimum salary and $82.9 \%$ lived with others. The MMRI showed that the family was the most important provider of care in all the dimensions assessed, but the elderly have their own small social networks. Elderly patients attended by the Family Health teams have low incomes and little formal education, and social support networks that are too small to meet their needs.

Key words Social support, Elderly, Family Health care
Resumo O envelhecimento tem especificidades marcadas pela posição de classe de indivíduos e grupos sociais, assim como pela cultura, política, condições socioeconômicas e sanitárias das coletividades. No atendimento às necessidades específicas dos idosos, os sistemas de suporte social são essenciais. O objetivo deste artigo é descrever o perfil sociodemográfico e a rede de suporte social do idoso assistido pela Estratégia Saúde da Família. Trata-se de um estudo transversal com idosos residentes em Dourados (MS). Utilizou-se questionário sociodemográfico e Mapa Mínimo de Relações do Idoso (MMRI). Foram entrevistados 503 idosos: $69,0 \%$ são do sexo feminino, 53,1\% são analfabetos, 58,3\% têm renda per capita inferior a um salário mínimo e 82,9\% moram acompanhados. $O$ MMRI destacou a família como maior provedora de cuidados em todas as dimensões avaliadas, mas os idosos possuem redes sociais pequenas. Os idosos assistidos pelas ESF têm baixa renda e escolaridade, e possuem uma rede de suporte social pequena para atender às suas necessidades.

Palavras-chave Apoio social, Idoso, Saúde da Família 


\section{Introdução}

O processo de envelhecimento é um fenômeno que percorre toda a história da humanidade, mas apresenta características diferenciadas de acordo com a cultura, o tempo e o espaço. O envelhecimento tem especificidades marcadas pela posição de classe de indivíduos e grupos sociais, assim como pela cultura, política, condições socioeconômicas e sanitárias das coletividades. As relações sociais podem ter um papel essencial para manter ou mesmo promover a saúde física e mental dos idosos. Os efeitos positivos do suporte social estão associados com a utilidade de diferentes tipos de suporte fornecidos pela família: emocional ou funcional ${ }^{1}$.

Matsukura et al. ${ }^{2}$ destacam a associação entre suporte social e níveis de saúde, da mesma forma que identificam a presença de serviços sociais funcionando como um ajuste "protetor" ante o risco de doenças induzidas por estresse ${ }^{2}$.

No atendimento às necessidades específicas dos idosos, os sistemas de suporte social são essenciais, sendo classificados em formais e informais. Entende-se por sistema formal os serviços de atendimento ao idoso que incluem hospitais, instituição de longa permanência (ILPI), atendimento domiciliar, programas formais de capacitação de pessoal voltados ao atendimento dessa população. Já o sistema informal ou rede de suporte social compreende as redes de relacionamentos entre membros da família, amigos, relações de trabalho, de inserção comunitária e de práticas sociais ${ }^{3,4}$.

A família tem especial relevância, por ser o contexto social mais próximo no qual os indivíduos estão envolvidos, e os relacionamentos mantidos por corresidentes em seus arranjos domiciliares têm implicações positivas para a saúde. Focase o arranjo domiciliar pela necessidade de conhecer o processo social pelo qual as novas estruturas familiares dos idosos demandam e fornecem sistemas de apoio formal e informal ${ }^{5}$.

A avaliação objetiva da composição familiar e das funções que seus membros exercem é importante porque fornece informações significativas para melhorar o planejamento do cuidado aos idosos, uma vez que a família satisfaz numerosas necessidades de seus componentes, sejam físicas (alimentação, habitação, cuidados pessoais), psíquicas (autoestima, amor, afeto) ou sociais (identificação, relação, comunicação, pertencimento a um grupo). O suporte social da família ajuda os idosos no processo de enfrenta- mento e recuperação de enfermidades, estresse e outras experiências difíceis da vida ${ }^{6}$.

Entrevistas com as famílias são fontes muito ricas em informações e propiciam maior interação entre os envolvidos. Alguns instrumentos vêm sendo desenvolvidos para avaliar a dinâmica de funcionamento familiar, de modo a subsidiar a proposição de intervenções que auxiliem o restabelecimento do equilíbrio dessa unidade de relações, sempre que uma disfunção é detectada.

A rede social pode ser avaliada quanto às características estruturais, funções dos vínculos e dos atributos de cada vínculo e pode ser registrada em forma de mapa de relações que inclui todos os indivíduos com quem uma determinada pessoa interage ${ }^{4}$. Entretanto, a revisão da literatura sobre escalas para avaliação do apoio social sugere que nenhuma é inteiramente adequada e que a confiabilidade, a validade e os fatores estruturantes dos instrumentos existentes são poucos conhecidos ${ }^{7}$.

\section{Metodologia}

Este artigo tem como objetivo descrever o perfil sociodemográfico e a rede de suporte social do idoso assistido pela Estratégia Saúde da Família. Realizou-se um estudo primário, observacional, do tipo transversal, com idosos residentes em Dourados (MS). O município de Dourados situa-se a $214 \mathrm{~km}$ da capital, Campo Grande, e os dados do Instituto Brasileiro de Geografia e Estatística (IBGE) de 2007 registraram uma população de 181.869 habitantes. O Departamento de Informática do Sistema Único de Saúde (Datasus) estimou que, em 2007, Dourados apresentava uma população idosa de 15.753 pessoas, correspondendo a $8,7 \%$ da população total ${ }^{8}$.

Em agosto de 2008, a Atenção Básica dispunha de três Unidades Básicas de Referência, 23 Unidades Básicas de Saúde da Família (UBSF) na área urbana e sete na zona rural, com 32 equipes de Estratégia Saúde da Família (ESF) na área urbana e cinco na rural. Existem cinco Estratégias de Agente Comunitário de Saúde no perímetro urbano e dois indígenas. As UBSF localizamse em bairros afastados do centro, cujos moradores são, principalmente, trabalhadores do setor agropecuário, do comércio e da indústria. Em algumas dessas áreas, predominam famílias com baixo poder aquisitivo. As equipes ESF e EACS de Dourados proporcionavam cobertura populacional de $65,04 \%$. 


\section{Sistema Municipal de Assistência Social}

A Secretaria Municipal de Assistência Social e Economia Solidária (Semases) foi criada em 2000, e as ações voltadas para a pessoa idosa são recentes. A Política Municipal da Pessoa Idosa foi promulgada em 29 de novembro de 2004, através da Lei no 2.717 , e a partir dessa data foi instituído o Conselho Municipal de Defesa da Pessoa Idosa $a^{9}$.

O município possui uma rede de suporte social reduzida ante a significativa demanda de idosos que necessitam de atenção especializada, tanto na área social quanto na de saúde. Em junho de 2008, a rede formal de atendimento e proteção social destinada aos idosos em Dourados era constituída por entidades governamentais e não governamentais, a saber:

. CRAS - Centro de Referência da Assistência Social: órgão público municipal que oferece serviços continuados de proteção básica de assistência social às famílias, grupos e indivíduos em situação de vulnerabilidade social ${ }^{10}$. Há cinco unidades no município, sendo quatro na zona urbana e uma na reserva indígena. Todas possuem o Programa de Atenção Integral à Família (PAIF), que promove o fortalecimento dos vínculos familiares e comunitários, por meio da atuação de equipes multiprofissionais.

. CREAS - Centro de Referência Especializado de Assistência Social: unidade pública municipal de prestação de serviços especializados e continuados a indivíduos e famílias com seus direitos violados $^{10}$. Há apenas um CREAS no município.

- Centro de Convivência do Idoso André Chamorro: administrado pelo município, atende pessoas com idade acima de 55 anos, de ambos os sexos, independentes fisicamente, com ações voltadas para a melhoria da qualidade de vida.

- Lar do Idoso: instituição de longa permanência, de natureza privada, com capacidade para atender cinquenta idosos.

- Pastoral do Idoso: composta por voluntários da Igreja Católica que fazem visitas a pessoas idosas, oferecendo ajuda material e auxiliando nos cuidados de higiene de idosos acamados.

\section{Sujeitos da pesquisa}

Foram pesquisados idosos residentes em Dourados (MS), cadastrados nas equipes de Estratégia Saúde da Família e selecionados por amostragem aleatória. Os critérios de inclusão foram: pessoas de ambos os sexos, com idade igual ou superior a 60 anos, tendo sido excluídos os idosos incapazes de se comunicar, os indígenas residentes nas aldeias e os que se recusaram a participar ou assinar o termo de consentimento livre e esclarecido.

Foram sorteados 672 idosos; destes, cinco recusaram-se a participar do estudo, 135 não se encontravam no domicílio por ocasião da coleta de dados e 29 foram excluídos pela incapacidade de se comunicar, resultando em 503 idosos participantes.

O tamanho da amostra foi calculado considerando-se $\mathrm{p}=0,5$, com intervalo de confiança igual a $95 \%$, para $25 \%$ de perdas e $4,4 \%$ de precisão, portanto (n) de 497 sujeitos.

A coleta de dados ocorreu no período de junho de 2007 a março de 2008. Das 32 equipes da ESF existentes na área urbana, 28 participaram do estudo, uma vez que nem todas estavam consolidadas.

As variáveis demográficas e socioeconômicas foram: idade (categorizada por faixa etária de 60 a 69, 70 a 79 e 80 anos ou mais); sexo; cor da pele definida pela autodenominação e classificada em branco, preto e pardo (que incluiu indígena e amarelo); escolaridade (analfabetos e alfabetizados); estado civil (solteiro, união consensual, viúvo e separados); arranjo familiar (mora sozinho ou acompanhado); número de filhos vivos; benefícios recebidos (aposentadoria, pensão, benefício de prestação continuada, outros, nenhum); renda per capita; número de pessoas que residem no domicílio.

A rede de suporte social foi avaliada pelo Mapa Mínimo de Relações do Idoso (MMRI), adaptado e modificado por Domingues ${ }^{11}$ do Mapa Mínimo de Relações de Sluzki, para poder identificar a rede de suporte social do idoso.

O MMRI é constituído por quatro quadrantes que representam família, amigos, comunidade e relações com os serviços sociais ou de saúde. Sobre esses quadrantes inscrevem-se três áreas, ou seja, um círculo interno de relações mais próximas, cujos contatos ocorrem pelo menos uma vez por semana; um círculo intermediário de relações pessoais com encontros que acontecem pelo menos uma vez por mês; um círculo externo de conhecimento e contatos ocasionais, no mínimo uma vez por ano.

Os relacionamentos significativos são dispostos nos círculos para simbolizar os diversos graus de proximidade de relacionamento. Para configurar um mapa de relações, é necessário descrever a proximidade do relacionamento segundo a percepção do pesquisado e a frequência com que o contato ocorre. 
O Mapa Mínimo de Relações do Idoso pode ser categorizado estruturalmente pelo número de pessoas que são efetivamente significativas para o idoso. Segundo Sluzki ${ }^{4}$, há indicativos de que as redes de tamanho médio são mais efetivas do que as pequenas ou muito numerosas.

O tamanho da rede de suporte social corresponde ao número de registros no MMRI, segundo a percepção do idoso. Além do tamanho, esse instrumento permite conhecer a amplitude dos relacionamentos significativos mencionados por quadrante, em termos de composição (membros da família e da comunidade), frequência de contatos e função desempenhada.

Seguindo a orientação de Domingues ${ }^{11}$, este estudo adotou o seguinte padrão: até duas pessoas no primeiro círculo caracterizam uma rede pequena; entre três e cinco pessoas no mesmo círculo, rede média; e acima de seis pessoas por círculo, rede grande. Este resultado foi obtido pela divisão do número de pessoas citadas no primeiro círculo pelo número de idosos pesquisados.

\section{Procedimentos éticos}

Os procedimentos realizados não apresentaram riscos ou prejuízos para os sujeitos da pesquisa. Foram cumpridas as exigências da Resolução no 196/96, e a participação de cada idoso foi autorizada pelo próprio ou seu responsável legal, por meio da assinatura do termo de consentimento livre e esclarecido. O Comitê de Ética da Escola de Enfermagem da Universidade de São Paulo aprovou o estudo, e a Secretaria Municipal de Saúde de Dourados autorizou a pesquisa.

\section{Resultados}

Foram entrevistados 503 idosos, sendo 156 $(31,0 \%)$ do sexo masculino e $347(69,0 \%)$ do feminino. A média de idade foi 72,6 anos $(\mathrm{dp}=8,0)$ para o sexo masculino e 70,9 anos $(\mathrm{dp}=7,9)$ para o feminino. Entre os $267(53,1 \%)$ analfabetos, foram encontrados $95(35,6 \%)$ homens e 172 $(64,4 \%)$ mulheres.

Predominaram idosos viúvos, dos quais $83,3 \%$ eram do sexo feminino. Entre os entrevistados, a média de filhos vivos foi de 5,2 ( $\mathrm{dp}=2,9)$. Verificou-se que $86(17,1 \%)$ moravam sozinhos, $161(32,0 \%)$ residiam com outra pessoa, 111 $(22,1 \%)$ com duas pessoas, $60(11,9 \%)$ com três pessoas, $45(8,9 \%)$ com quatro pessoas e 40 $(8,0 \%)$ com cinco ou mais pessoas. Quanto à renda per capita, 167 (33,2\%) recebiam em média $\mathrm{R} \$$
200,00 ou menos por mês por pessoa (equivalente a $0,52 \%$ do SM [o salário mínimo vigente na época da coleta dos dados era de $\mathrm{R} \$ 380,00]), 126$ $(25,0 \%)$ entre $\mathrm{R} \$ 201,00$ e R $\$ 370,00$ por mês (de $0,53$ a $0,97 \%$ do $S M)$ e 210 ( $41,7 \%)$, mais de $R \$$ 370,00 por pessoa por mês $(0,98 \%$ do SM). As demais características sociodemográficas e econômicas encontram-se dispostas na Tabela 1.

\section{Mapa Mínimo das Relações do Idoso (MMRI)}

Os indicadores de suporte social investigados mostraram uma importante participação da família e da comunidade no cuidado aos idosos, verificada nas várias modalidades de apoio que recebem (visita, companhia, limpeza da casa, cuidados pessoais e apoio financeiro).

Relativamente à primeira questão do MMRI quais as pessoas que o(a) visitam pelo menos: uma vez por semana (frequentemente), uma vez por mês (pouco frequentemente) e uma vez por ano (raramente)? -, no primeiro círculo de proximidade,

Tabela 1. Distribuição dos idosos atendidos por equipes da Estratégia Saúde da Família, segundo características demográficas e socioeconômicas Dourados (MS), 2008.

\begin{tabular}{lrr}
\hline \multicolumn{1}{c}{ Variáveis } & N & $\%$ \\
\hline Estado civil & & \\
$\quad$ Viúvo & 216 & 42,9 \\
Casado/união consensual & 209 & 41,6 \\
$\quad$ Separado/divorciado & 64 & 12,7 \\
$\quad$ Solteiro & 14 & 2,8 \\
Faixa etária & & \\
60-69 anos & 233 & 46,3 \\
70-79 anos & 191 & 38,0 \\
80 anos ou mais & 79 & 15,7 \\
Cor da pele & & \\
$\quad$ Branca & 365 & 72,6 \\
Parda & 82 & 16,3 \\
$\quad$ Preta & 56 & 11,1 \\
Escolaridade & & \\
$\quad$ Analfabeto & 267 & 53,1 \\
$\quad$ Alfabetizado & 236 & 46,9 \\
Arranjo familiar & & \\
$\quad$ Acompanhado & 417 & 82,9 \\
$\quad$ Sozinho & 86 & 17,1 \\
Vínculo econômico do inativo & & \\
Aposentado & 273 & 54,3 \\
Pensionista & 113 & 22,5 \\
Outros & 41 & 8,1 \\
$\quad$ Nenhum & 76 & 15,1 \\
Total & $\mathbf{5 0 3}$ & $\mathbf{1 0 0}$ \\
\hline
\end{tabular}


das visitas semanais, a participação da família foi de $69,2 \%$, seguida pelos membros da comunidade $(17,1 \%)$, amigos $(8,3 \%)$ e serviço social e de saúde $(5,4 \%)$, representado majoritariamente pelos agentes comunitários de saúde (ACS).

O tamanho da rede social dos idosos entrevistados foi considerado médio (média de 3,8 com $\mathrm{dp}=2,7$ e mediana $=3,0)$, composto por três a cinco pessoas, sendo que $31(6,2 \%)$ idosos não recebiam nenhuma visita na semana, $154(30,6 \%)$ possuíam rede social de tamanho pequeno, 197 (39,2\%) com rede de tamanho médio e 121 (24,0\%) tinham rede social de tamanho grande. O número máximo de contatos semanais registrado foi de 17 .

Por ordem de frequência, a rede de contatos sociais incluiu: filhos com uma participação de $48,8 \%$, netos com $12,4 \%$, outros parentes (noras, genros, sobrinhos) com 5,8\% e irmãos com $2,2 \%$ de participação. Os vizinhos e amigos respondem com $15,4 \%$ e $8,3 \%$, respectivamente. Os ACS representaram 5,4\%, e o restante ficou distribuído entre os grupos religiosos e cônjuges.

No círculo intermediário, a presença da família continuou sendo a mais freqüente, seguida pelo serviço social e de saúde, comunidade e amigos $(42,7 \%, 35,9 \%, 14,6 \%$ e $6,8 \%$, respectivamente). Entretanto, no quadrante representado pelos serviços social e de saúde, os ACS foram os que mais visitaram os idosos.

No último círculo, destacou-se a presença da família, com 60,8\%; dos profissionais de saúde (médicos, enfermeiras, auxiliares de enfermagem e dentistas), que representaram $33,5 \%$ dos contatos; da comunidade e dos amigos, com $4,5 \%$ e $1,2 \%$, respectivamente. Não houve referências aos profissionais do serviço social do município.

A segunda pergunta - com quem o(a) senhor(a) pode contar se desejar ou precisar de alguém para fazer-lhe companhia, pelo menos: uma vez por semana (frequentemente), uma vez por mês (pouco frequentemente), e uma vez por ano (raramente)? - apresentou menor número de participantes, porém, em todos os círculos, destacouse a presença maciça da família $(96,4 \%, 88,9 \%$ e $100,0 \%$, respectivamente na semana, no mês e no ano). O tamanho dessa rede foi considerado pequeno, uma vez que a média de pessoas que faziam companhia aos idosos foi de $2,3(\mathrm{dp}=1,6$ e mediana $=2,0)$. O número mínimo de contatos foi zero, no caso de 21 idosos (4,2\%); 308 (61,1\%) idosos possuem rede de suporte de tamanho pequeno; $152(30,2 \%)$ idosos têm rede de tamanho médio; e $22(4,4 \%)$ idosos têm a rede social com mais de seis componentes. O máximo encontrado foi de 11 pessoas por idoso.
No círculo intermediário, a presença da família é de $88,9 \%$, sendo os filhos os mais presentes $(42,6 \%)$, seguidos de netos $(23,2 \%)$, cônjuges $(17,0 \%)$, outros parentes $(11,6 \%)$ e irmãos $(2,0 \%)$, porém $482(95,8 \%)$ idosos referiram não poder contar com nenhuma pessoa para lhe fazer companhia, pelo menos uma vez por mês. $\mathrm{O}$ mesmo ocorreu no último círculo, em que 21 idosos $(4,2 \%)$ podem contar com pelo menos uma pessoa, por ano, para lhe fazer companhia.

A questão três - a quem o(a) senhor(a) recorre ou recorreria se precisar de ajuda para cuidar das coisas da casa, como por exemplo arrumar, limpar, cozinhar ou fazer compras, pelo menos: uma vez por semana (frequentemente), uma vez por mês (pouco frequentemente) e uma vez por ano (raramente)? revelou uma rede pequena, com média de 1,6 pessoas $(\mathrm{dp}=1,1$ e mediana=1,0). O mínimo de contatos foi zero para 40 (8,0\%) idosos; 394 (78,3\%) idosos possuem uma rede social pequena; 63 $(12,5 \%)$ têm uma rede social de tamanho médio; e apenas seis $(1,2 \%)$ possuem rede social grande.

A família continua sendo a rede de suporte social mais freqüente, e as filhas $(39,3 \%)$, netas $(10,2 \%)$, esposas e noras (ambas com 9,6\%) são as maiores responsáveis por esse tipo de auxílio. A presença masculina também fez-se presente, embora em menor número, representada por maridos $(5,0 \%)$, filhos $(9,6 \%)$, netos $(2,5 \%)$ e outros parentes $(2,2 \%)$. Dos membros da comunidade, destacou-se a presença de empregadas domésticas $(6,1 \%)$, vizinhas $(4,1 \%)$, membros do grupo religioso $(0,3 \%)$ e amigas $(1,5 \%)$.

Já no círculo intermediário, 474 (94,2\%) idosos não têm ninguém para ajudá-los nos serviços da casa, e 495 (98,4\%) idosos referem o mesmo no último círculo.

A pergunta quatro - a quem o(a) senhor(a) recorre ou recorreria se precisar de ajuda para os cuidados pessoais, como por exemplo trocar de roupa, tomar banho, comer, se levantar, se deitar, pelo menos: uma vez por semana (frequentemente), uma vez por mês (pouco frequentemente) e uma vez por ano (raramente)? - ambém evidenciou uma rede de suporte social pequena, com média de 1,6 pessoas $(\mathrm{dp}=1,2$ e mediana $=1,0)$. Para 56 $(11,1 \%)$ idosos, não há nenhuma pessoa com quem se possa contar formal ou informalmente; $359(71,4 \%)$ idosos têm uma rede social pequena; $85(16,9 \%)$ possuem rede social tamanho médio; e três $(0,6 \%)$ têm rede social grande.

Verificou-se que, para esses idosos, a família ainda é a maior provedora de cuidados, principalmente os filhos de ambos os sexos $(63,6 \%)$, dentre os quais sobressaem as filhas $(46,8 \%)$. As 
esposas aparecem com $8,6 \%$, e os maridos com $4,4 \%$. A participação das netas $(4,5 \%)$ é o dobro da dos netos. Outros parentes representam $8,6 \%$, e juntos, irmãs e irmãos perfazem 1,4\% (sendo a presença das irmãs de 1,2\%). As vizinhas representaram 3,9\% e as prestadoras de serviços, somente $1,5 \%$. Também foram mencionados amigos $(0,8 \%)$, membros de grupos religiosos $(0,3 \%)$ e $\operatorname{ACS}(0,2 \%)$.

No círculo intermediário, foram identificados cinquenta $(9,9 \%)$ idosos que continuam contando com o apoio da família, principalmente dos filhos $(73,8 \%)$. No último círculo, apenas dez idosos referiram que podem contar com um filho ou filha, pelo menos uma vez por ano, para ajudar nos cuidados pessoais.

A última questão - quem ajuda ou ajudaria o(a) senhor(a) financeiramente se precisar de auxílio para pagar aluguel da casa, pagar uma conta, comprar comida, remédio, etc., pelo menos: uma vez por semana (frequentemente), uma vez por mês (pouco frequentemente) e uma vez por ano (raramente)? - confirmou a família como a principal rede de apoio em todos os círculos de proximidade.

O tamanho da rede de auxílio financeiro também foi considerado pequeno, uma vez que a média foi de 1,2 pessoas $(\mathrm{dp}=1,2$ e mediana $=1,0)$. Para $163(32,4 \%)$ idosos não existe rede de suporte social na semana; $274(54,5 \%)$ têm rede social pequena; $62(12,3 \%)$ possuem rede de tamanho médio; e quatro $(0,8 \%)$ idosos têm rede social grande. Esse auxílio provinha principalmente de filhos $(76,5 \%)$, cônjuges $(8,2 \%)$, outros parentes $(5,7 \%)$, netos $(3,6 \%)$ e irmãos $(1,6 \%)$. Os vizinhos representaram $2,9 \%$; os amigos, $1,1 \%$; e os grupos religiosos, $0,3 \%$.

Alguns idosos justificaram que os filhos ganhavam pouco ou que moravam longe, por isso tinham dificuldades para ajudá-los, observação que revela o baixo poder aquisitivo familiar. Outros diziam que só podiam contar com Deus.

No círculo intermediário, 415 idosos $(82,5 \%)$ não podem contar com ninguém para auxílio financeiro. E entre os 88 (17,5\%) idosos que recebem apoio financeiro, os filhos foram responsáveis por $85,2 \%$ da ajuda oferecida. Como apoio formal, uma idosa declarou obter ajuda simultânea da Rede Feminina de Combate ao Câncer e da Secretaria de Assistência Social e Economia Solidária de Dourados, da qual recebia uma cesta básica de alimentos.

No último círculo, 402 (79,9\%) idosos não contam com nenhuma ajuda, e o empréstimo consignado (sistema formal) foi citado por 23 pessoas $(15,4 \%)$.

\section{Discussão}

\section{Perfil sociodemográfico}

As características sociodemográficas dos idosos entrevistados assemelham-se às tendências observadas nos estudos populacionais brasilei$\operatorname{ros}^{12-14}$. A maioria dos entrevistados declarou-se branca, católica participante de cultos semanais, com baixa escolaridade, baixa renda e pouca participação em atividades sociais em associações comunitárias ou clubes de lazer. Houve acentuado predomínio do sexo feminino, com grande proporção de viúvas, caracterizando a feminização do envelhecimento, consequência da sobremortalidade masculina.

Observou-se ainda grande número de filhos. Para Saad ${ }^{15}$, muitos filhos podem expressar uma rede potencial de apoio social informal. Estudo de Bini (realizado em Santa Bárbara do Sul, RS) ${ }^{16}$ constatou que os idosos entrevistados haviam constituído famílias numerosas, pois 39,4\% referiram seis ou mais filhos. Pilger ${ }^{17}$, em estudo desenvolvido em Veranópolis (RS), também constatou que 38,4\% dos entrevistados haviam tido seis filhos ou mais, com média de cinco filhos vivos. No município de São Paulo, o Projeto Saúde, Bem-Estar e Envelhecimento (SABE) identificou que $32 \%$ dos idosos tinham de três a quatro filhos vivos, e $25 \%$, cinco ou mais filhos ${ }^{15}$.

A convivência familiar é visível tanto em Dourados, onde $83 \%$ dos entrevistados moram acompanhados, quanto em Porto Alegre (RS) (78,1\%), São Paulo (SP) (87,0\%), Santa Bárbara do Sul (RS) $(71,2 \%)$, Veranópolis (RS) (53,9\%) e Bambuí (RS) $(84,1 \%)$. Na Espanha, apenas $14,2 \%$ dos idosos com idade igual ou superior a 65 anos vivem sozinhos, com destaque para as mulheres $(19,6 \%)$ em relação aos homens $(6,9 \%)^{14-19}$.

Em 2003, a proporção dos idosos brasileiros que não sabiam ler ou escrever era de aproximadamente quatro milhões, ou seja, 1/3 dessa parcela populacional. Em 2000, quando a média de anos de estudos para os idosos brasileiros era de 3,4, o Estado de Mato Grosso do Sul registrou média de 2,8 anos, inferior à nacional ${ }^{20}$. Em 2000, MS tinha 36\% dos idosos como analfabetos, sendo que a taxa era maior entre as mulheres: 40,2\% contra $32 \%$ do sexo masculino ${ }^{21}$.

No Brasil, até a década de 50 do século passado, a educação era considerada privilégio de poucos, uma vez que, culturalmente, cabia aos homens o sustento do lar, e às mulheres, a responsabilidade de cuidar da casa e dos filhos. Considerava-se que não havia a necessidade de as 
mulheres aprenderem a ler e escrever. Outro fator determinante para o analfabetismo era a dificuldade de acesso à escola, principalmente no meio rural, uma vez que as escolas localizavam-se nos povoados ou nas vilas mais próximas ${ }^{16}$.

Os rendimentos dos idosos pesquisados mostraram-se semelhantes aos ganhos da população de Dourados. Em 2000, informações sobre as classes de rendimento dos residentes no município registraram que, de um total de 131.949 habitantes com idade superior a 10 anos, $39,9 \%$ não possuíam rendimentos, enquanto $16,5 \%$ recebiam até um salário mínimo e 18,3\%, entre um e dois salários ${ }^{21}$.

Dentre os idosos brasileiros, predominam os aposentados, com baixa renda mensal e per capi$\mathrm{ta}$, sendo que as mulheres possuem renda inferior à dos homens. Segundo o IBGE, a participação dos idosos responsáveis por domicílios está aumentando, principalmente nos estratos de renda mais baixos, pois eles dão suporte material e financeiro para os demais membros da família, como filhos adultos e netos ${ }^{13,14,16,20}$.

Em 2003, a maior parte da renda dos idosos brasileiros, homens ou mulheres, provinha da Seguridade Social, ou seja, de aposentadorias, pensões ou benefícios da Assistência Social.

Para Soares et al. ${ }^{22}$, houve redução da desigualdade de renda no Brasil entre 1995 e 2004, com diminuição da pobreza, em decorrência dos programas de transferência de renda, tais como o Bolsa Família e o Benefício de Prestação Continuada.

\section{Rede de suporte social}

Há estudos evidenciando que diferenças na estrutura das relações sociais em decorrência de diferenças de idade, sexo, estado civil, renda per capita, escolaridade e autopercepção de saúde afetam a estrutura e o tipo de apoio social entre os idosos entrevistados ${ }^{23,24}$.

Neste estudo, os achados permitiram identificar que a rede de suporte social dos idosos de Dourados é pequena na maioria das dimensões avaliadas (companhia, cuidados pessoais e da casa e financeiro) e que o sistema provedor mais presente é o familiar.

Estudo utilizando o questionário Medical $\mathrm{Ou}$ tcomes Study foi desenvolvido no município de Taquarituba (SP), com o propósito de descrever as características de apoio social aos idosos residentes em área rural e assistidos pela ESF. Os resultados permitiram constatar que quanto maior o número de pessoas no domicílio, maior a frequência de apoio afetivo. Idosos com maior probabili- dade de apresentar alguma inadequação de apoio social predominam entre as mulheres, os analfabetos, os viúvos ou solteiros e os de menor renda ${ }^{25}$.

A avaliação da estrutura e das funções de redes de apoio social de idosos mexicanos, por meio da Escala de Bem-Estar Subjetivo, também constatou que a família (filhos e cônjuge), amigos e vizinhos são os provedores mais frequentes (semanalmente) de cuidados. Em seguida, apareceram os membros de grupos religiosos e, por último, os profissionais de saúde. O suporte social formal (profissionais de saúde) foi mais frequente quando avaliado pelo menos alguns dias do mês. Quanto à função de apoio para ajudar a resolver os problemas do dia a dia, 35\% dos idosos recebiam-na dos seus cônjuges, 30,6\% dos filhos e $20 \%$ relatavam não ter ninguém. Em relação à ajuda financeira, 43,8\% dos entrevistados contavam com os filhos, $18,1 \%$ com o cônjuge e $28,1 \%$ não tinham com quem contar. Para acompanhálos a algum lugar, 37,5\% dependiam dos filhos, 25,6\% dos cônjuges e 15,6\% não possuíam ninguém para essa função ${ }^{26}$.

Rosa et.al. ${ }^{24}$ utilizaram os índices de frequência e diversidade de contatos, o status de coabitação e a situação conjugal para investigar as redes sociais e a disponibilidade de apoio social instrumental e informal de 1.568 idosos no município de São Paulo. O estado civil influenciou o tamanho da rede, pois mulheres e homens solteiros, assim como os homens mais pobres, apresentavam frequências de contatos mais baixas.

\section{Conclusões}

Esta investigação permitiu identificar o perfil e a rede social de apoio de idosos assistidos na Atenção Básica por equipes da ESF no município de Dourados. A maioria dos entrevistados era do sexo feminino, viúvos, de baixo poder aquisitivo, que conviviam com duas ou três gerações. Sua rede de suporte social era composta predominantemente pela família, comunidade e amigos. A participação da rede formal é incipiente. Os serviços de saúde foram representados pelos ACS e o serviço social esteve ausente.

Sabe-se que os profissionais da saúde, especialmente aqueles que atuam na Atenção Básica, necessitam de instrumentalização sistematizada para direcionar seu olhar para além dos indivíduos, buscando compreender a funcionalidade familiar como um componente essencial do planejamento assistencial para o alcance do sucesso terapêutico. 
O Mapa Mínimo de Relações do Idoso revelou-se um instrumento de fácil aplicação, passível de utilização na Atenção Básica. Espera-se que os resultados deste estudo ofereçam subsídios para a elaboração de protocolos de avaliação de necessidades sociais de idosos, nesse nível de atenção.

\section{Colaboradores}

MRM Alvarenga, MAC Oliveira, MA Domingues, F Amendola e O Faccenda participaram igualmente de todas as etapas da pesquisa e da elaboração do artigo.

\section{Referências}

1. Ramos MP. Apoio social e saúde entre idosos. Sociologias 2002; 4(7):156-175.

2. Matsukura TS, Marturano EM, Oishi J. O Questionário de Suporte Social (SSQ): estudos da adaptação para o português. Rev Lat Am Enferm 2002; 10(5):675-681.

3. Lemos N, Medeiros SL. Suporte social ao idoso dependente. In: Freitas EV, Py L, Cançado FAX, Gorzoni ML, organizadores .Tratado de geriatria e gerontologia. Rio de Janeiro: Koogan; 2006. p. 892-897.

4. Sluzki CE. A rede social na prática sistêmica: alternativas terapêuticas. São Paulo: Casa do Psicólogo; 1997.

5. Rosa TEC. Determinantes do estado nutricional de idosos do município de São Paulo: fatores socioeconômicos, redes de apoio social e estilo de vida [tese]. São Paulo: Faculdade de Saúde Pública da Universidade de São Paulo; 2005.

6. Moragas RM. Gerontologia social: envelhecimento e qualidade de vida. 2a ed. São Paulo: Paulinas; 2004.

7. Clemente MA. Redes sociales de apoyo en relación al proceso de envejecimiento humano: revisión bibliográfica. Interdisciplinaria - Rev Psicol Cienc Afines 2003; 20(1):31-60.

8. Brasil. Ministério da Saúde. Datasus [homepage na Internet]. Brasília; 2008. [acessado 2008 maio 30]. Disponível em: http://w3.datasus.gov. br/datasus/ datasus.php

9. Mato Grosso do Sul. Dourados. Prefeitura de Dourados. Lei no 2.717, de 29 de novembro de 2004. Dispõe sobre a Política Municipal da Pessoa Idosa. Dourados: Prefeitura de Dourados; 2004. [texto na internet]. 2004[acessado 2008 mar 28]. Disponível em: htpp://www.dourados.ms.gov.br

10. Brasil. Ministério do Desenvolvimento Social e Combate à Fome. Secretaria Nacional de Assistência Social. Centro de Referência Especializado de Assistência Social: guia de orientação. Brasília: Ministério do Desenvolvimento Social e Combate à Fome; 2009. 
11. Domingues MARC. Mapa mínimo de relações: instrumento gráfico para identificar a rede de suporte social do idoso [tese]. São Paulo: Faculdade de Saúde Pública da Universidade de São Paulo; 2004.

12. Pereira RS, Curioni CC, Veras R. Perfil demográfico da população idosa no Brasil e no Rio de Janeiro em 2002. Texto sobre Envelhecimento 2003; 1(6):43-59.

13. Jóia LC, Ruiz T, Donalísio MR. Grau de satisfação com a saúde entre idosos do município de Botucatu, Estado de São Paulo, Brasil. Epidemiol Serv saúde 2008; 17(3):187-194.

14. Paskulin LMG, Vianna LAC. Perfil sociodemográfico e condições de saúde auto-referidas de idosos de Porto Alegre. Rev Saude Publica 2007;41(5):757-768.

15. Saad PM. Arranjos domiciliares e transferências de apoio informal. In: Lebrão ML, Duarte YAO. SABE - Saúde, Bem-Estar e Envelhecimento: o Projeto SABE no município de São Paulo: uma abordagem inicial. Brasília: Organização Pan-Americana da Saúde; 2003. p. 203-210.

16. Bini R. Estudo multidimensional sobre a população de idosos do município de Santa Bárbara do Sul [dissertação]. Porto Alegre: Pontifícia Universidade Católica do Rio Grande do Sul; 2006.

17. Pilger JW. Perfil do idoso atendido por um Programa de Saúde da Família em Veranópolis, RS [dissertação]. Porto Alegre: Pontifícia Universidade Católica do Rio Grande do Sul; 2006.

18. Lima Costa MF, Guerra HL, Firmo JOA, Uchoa E. Projeto Bambuí: um estudo epidemiológico de características sociodemográficas, suporte social e indicadores de condição de saúde dos idosos em comparação aos adultos jovens. Inf Epidemiol SUS 2001; 10(4):147-161.

19. Ortiz LP. Indicadores sociales. In: Ortiz LP, editor. Envejecer en España: II Asamblea Mundial sobre el Envejecimiento. Madrid: IMSERSO; 2002.

20. Camarano AA. Idosos brasileiros: indicadores de condições de vida e de acompanhamento de políticas. Brasília: Presidência da República, Subsecretaria de Direitos Humanos; 2005. p. 17-24.
21. Mato Grosso do Sul. Secretarias de Estado do Meio Ambiente, das Cidades, do Planejamento, da Ciência e Tecnologia. Superintendência de Planejamento. Indicadores básicos de Mato Grosso do Sul. Campo Grande: Banco de Dados do Estado - BDE/MS; 2007. [texto na Internet]. [acessado 2008 maio 15]. Disponível em: http://www.semac.ms.gov.br; http:/ /tabnet.datasus.gov.br

22. Soares FV, Soares S, Medeiros M, Osório RG. Programas de transferências de renda no Brasil: impacto sobre a desigualdade. Brasília: Ipea; 2006. (Texto para discussão, n. 1228).

23. Due P, Holstein B, Lund R, Modvig J, Avlund K. Social relations: network, support and relation strain. Social Science \& Medicine 1999; 48:661-673.

24. Rosa TEC, Benício MHDA, Alves MCGP, Lebrão ML. Aspectos estruturais e funcionais do apoio social de idosos do município de São Paulo, Brasil. Cad Saude Publica 2007; 23(12):2982-2992.

25. Pinto JLG, Garcia ACO, Bocchi SCM, Carvalhaes MABL. Características de apoio social oferecido a idosos de área rural assistida pelo PSF. Cien Saude Colet 2006; 11(3):753-764.

26. Noriega JAV, Quinones TIS, Guedea MTD. Bienestar subjetivo, enfrentamiento y redes de apoyo social en adultos mayores. Rev Intercont Psicol Educ 2005; 7(2):57-78.

Artigo apresentado em 25/11/2008

Aprovado em 08/06/2009

Versão final apresentada em 09/07/2009 